\title{
ENERGY DEPOSITION BY DYNAMICAL PAIR PRODUCTION IN ULTRARELATIVISTIC COLLISIONS
}

\author{
Cécile MARTIN, Dominique VAUTHERIN \\ Division de Physique Théorique '. Institut de Physique Nucléaire, F-91406 Orsay Cedex, France
}

and

Joseph CUGNON

Institui de Physique B5, Université de Liège au Sart-Tilman, B-4000 Liege J, Belgium

Received 6 March 1989; revised manuscript received 9 May 1989

\begin{abstract}
We investigate a mechanism of energy deposition through dynamical pair production in ultrarelativistic collisions. This mechanism arises because of the time dependence of the color charges created at the early stage of the reaction. We argue that such a mechanism may become comparable with that of Schwinger at high energies (about $1 \mathrm{TeV}$ per nucleon CM). We discuss the production rates of heavy flavors and their energy dependence. We point out that this mechanism should be kept in mind when discussing signatures of a quark-gluon plasma formation.
\end{abstract}

An important issue in the study of ultrarelativistic nuclear collisions is to determine whether the energy density reached during the reaction is high enough to lead to the formation of a quark-gluon plasma and to allow an exploration of the phase diagram of matter at high excitation. In this context a particularly useful approach has been developed, namely the flux tube model. This model was introduced by Low and Nussinov $[1,2]$ to describe hadron-hadron collisions, and later on extended to nuclear collisions by Biro, Nielsen and Knoll [3] and Gatoff, Kerman, Matsui and Svetitsky $[4,5]$ who have shown that the model is able to describe quantitatively a large variety of experimental data.

An attractive feature of the model is that it provides a detailed scenario of the reaction. At the early stage of the collision gluon exchanges between the nuclei lead to the formation of chromoelectric flux tubes as the nuclei recede. The strong chromoelectric field which was produced eventually decays by the Schwinger mechanism $[6,7]$ i.e. production of quark-

1 Unité de Recherche des Universités Paris XI et Paris VI associée au CNRS. antiquark pairs out of the vacuum. The corresponding pair production rate $\mathrm{W}$ is generally calculated by means of Schwinger's formula,

$W=\frac{g^{2} E^{2}}{4 \pi^{2}} \sum_{n \geqslant 1} \frac{1}{n^{2}} \exp \left(-\frac{n \pi m^{2}}{g|E|}\right)$,

which corresponds to the case of a static uniform field extending to infinity. In eq. (1) $E$ is the field strength, $m$ the mass of the created particle, $g$ their coupling constant to the field. The $E$ dependence of this formula reflects the nonperturbative nature of Schwinger's mechanism, which can be interpreted and calculated as a tunneling through a linear potential barrier [8].

Schwinger's mechanism was found in ref. [5] to be a quite efficient mechanism of energy deposition since it is able to turn into pairs about $20 \%$ of the field energy. In recent articles however it was shown that Schwinger's production rate may be significantly reduced by finite-size effects $[9,10]$. This makes it worthwhile to also consider an alternative mechanism which is simply the perturbative excitation of pairs out of the vacuum due to the time dependence of the chromoelectric field. It is the purpose of this 
present paper to investigate the importance of this effect and to work out whether it can provide a significant contribution to the energy deposited in ultrarelativistic collisions.

The total number $N$ of pairs produced and the associated variation $\Delta E$ of the vacuum energy by a time dependent external color field $A_{i i}(x)$ are determined to lowest order in the QCD coupling constant $\alpha_{s}=g^{2} /$ $4 \pi$ from the transition amplitude $\langle 0|S| \mathrm{q} \overline{\mathrm{q}}\rangle$ between the quark vacuum $|0\rangle$ and a state $|q \bar{q}\rangle$ with one quark q and an antiquark $\overline{\mathrm{q}}$. the $S$ matrix is

$S=\mathrm{T} \exp \left(\mathrm{i} g \int \mathrm{d}^{4} x \sum_{a} \bar{\psi}(x) \frac{\lambda^{a}}{2} \gamma^{\mu} \psi(x) A_{\mu}^{a}(x)\right)$,

where the matrices $\lambda^{a} / 2$ are the $\mathrm{SU}(3)$ generators satisfying

$\operatorname{Tr}\left(\frac{\lambda^{a}}{2} \frac{\lambda^{h}}{2}\right)=\frac{n_{\mathrm{F}}}{2} \delta^{a h}$

where the trace is over color and flavor variables and where $n_{\mathrm{F}}$ is the number of quark flavors [8].

For an external field $A_{\mu}(x)$ in a single color direction, we can use the formulae in chapter 4 of ref. [8]. We then obtain the following expressions:

$$
\begin{aligned}
N & =\frac{n_{\mathrm{F}}}{2} \int \mathrm{d}^{4} q \theta\left(1-4 m^{2} / q^{2}\right) \operatorname{Im}(I(q)) \\
& \times\left(|\boldsymbol{E}(q)|^{2}-|\boldsymbol{B}(q)|^{2}\right), \\
\Delta E & =\frac{n_{\mathrm{F}}}{2} \int \mathrm{d}^{4} q \theta\left(1-4 m^{2} / q^{2}\right) 2 \theta\left(q^{0}\right) \operatorname{Im}(\Pi(q)) \\
& \times\left(|\boldsymbol{E}(q)|^{2}-|\boldsymbol{B}(q)|^{2}\right) .
\end{aligned}
$$

In these expressions $\operatorname{Im}(\Pi(q))$ is the imaginary part of the color response function (or the absorptive part of the vacuum polarization in the lowest order approximation ) [8]

$$
\operatorname{Im}(\Pi(q))=\frac{1}{3} \alpha_{\mathrm{s}}\left(1-4 m^{2} / q^{2}\right)^{1 / 2}\left(1+2 m^{2} / q^{2}\right),
$$

and $\boldsymbol{E}(q), \boldsymbol{B}(q)$ denote respectively the Fourier transforms of the electric and magnetic fields

$E(q)=\frac{1}{4 \pi^{2}} \int \mathrm{d}^{4} x E(x) \exp (\mathrm{i} q x)$,

with a similar formula for $\boldsymbol{B}$.

Note that the number of pairs $N$ is a Lorentz invar- iant quantity. Note also that because of the step function in eqs. (4), (5), the pair production rate vanishes when $q_{0}^{2}-q^{2}<4 m^{2}$. This is the case for a constant uniform field, for which $E(q) \simeq \delta^{4}(q)$ or a static field, or a moving charge with constant velocity, or two moving capacitor plates with constant velocities. However, $N$ and $\Delta E$ do not vanish in the case of two color charges created as a result of gluon exchanges during a collision of two nuclei. Even if the nuclei are assumed to have a constant velocity, the time dependence of the color charge produced may generate high enough frequencies in $E$ to create quark-antiquark pairs.

In order to determine the importance of this effect we will work out first the case of a flux tube with a radius $R$, whose extremities are located at $\pm v t$ along the $z$-axis. We assume that the color charge density $\sigma$ at the extremities has been created at time zero with a time dependence of the form

$\sigma(\rho, t)=\sigma_{0}(\rho) \int_{-\infty}^{+\infty} \theta(t) F\left(t-t^{\prime}\right) \mathrm{d} t^{\prime}$,

where $p=\left(x^{2}+y^{2}\right)^{1 / 2}$ is the distance to the collision axis and $F$ a form factor with a width $\tau$ e.g.

$F(t)=\frac{1}{\tau \sqrt{2 \pi}} \exp \left(-t^{2} / 2 \tau^{2}\right)$.

This factor incorporates the fact that it takes a time $\tau$ (which is of the order of the collision time) to build up the color charge via gluon exchanges.

In eq. (8), the radial dependence $\sigma_{0}(\rho)$ of the color charge distribution is conveniently taken to be also of gaussian form

$\sigma_{0}(\rho)=\sigma_{0} \exp \left(-\rho^{2} / R^{2}\right)$.

Other choices of the form factors $F(t)$ and $\sigma_{0}(\rho)$, such as a lorentzian shape for $F(t)$ and a uniform distribution for $\sigma(\rho)$ can also be worked out with similar results but more complicated formulae.

In order to obtain the total energy deposited between times $t=0$ and $t=T$ we now apply eqs. (5)(7). These equations actually correspond to the case $t=\infty$. However, the case $t=T$ is merely obtained by inserting a step function $\theta(t)-\theta(T-t)$ in the integrand of eq. (7). Combining (8)-(10) and (7) we obtain 


$$
\begin{aligned}
& |\boldsymbol{E}(q)|^{2}=\frac{1}{4 \pi} \sigma_{0}^{2} v T R^{4} \frac{\delta\left(q_{z}\right)}{q_{0}^{2}} \\
& \quad \times \exp \left[-\left(q_{x}^{2}+q_{y}^{2}\right) R^{2} / 2\right] \exp \left(-q_{0}^{2} \tau^{2}\right) .
\end{aligned}
$$

In deriving this equation we have assumed $T$ to be large in order to be able to replace the result of the time integration in eq. (7) by $4 \pi v T \delta\left(q_{z}\right) / q_{0}^{2}$. Furthermore, we eliminated the functions $\delta\left(q_{0} \pm v q_{z}\right)$ which do not contribute to the integral in eqs. (4), (5).

Let us consider two flavors of massless quarks. Inserting (11) into (5), setting $m=0$ and dividing $\Delta E$ by the volume $V=\pi R^{2} \times 2 v T$ of the tube, we find that the energy density produced by the time-dependence of the chromo-electric field is

$\mathscr{H}=\frac{\alpha_{\mathrm{s}}}{12 \pi} \sigma_{0}^{2} \ln \left(1+R^{2} / 2 \tau^{2}\right)$.

The fraction of the field energy density at the center $\sigma_{0}^{2} / 2$ transformed into pairs is thus

$x=\frac{\alpha_{\mathrm{s}}}{6 \pi} \ln \left(1+R^{2} / 2 \tau^{2}\right)$.

In actual collisions, fluctuations in color orientation occur with a coherence length of the order of the size of a nucleon. As a result the exchange of soft gluons occurring at the beginning of the collision is believed to lead to the formation of a color rope [3] i.e. an assembly of parallel independent flux tubes with a radius of about $1 \mathrm{fm}$. Let us now calculate the energy density reached near the collision axis for a central collision. In this case one has

$2 \tau=\frac{1}{v_{\mathrm{P}}+v_{\mathrm{T}}}\left(\frac{2 R_{\mathrm{T}}}{\gamma_{\mathrm{T}}}+\frac{2 R_{\mathrm{P}}}{\gamma_{\mathrm{P}}}\right)$.

In eq. (14) the indices $\mathrm{P}$ and $\mathrm{T}$ refer to the projectile and target respectively and the $v$ 's are the velocities in the center of mass frame, $R$ the nuclear radius and $\gamma$ the usual notation for $1 / \sqrt{1-v^{2} / c^{2}}$. For identical nuclei and eq. (14) gives $c \tau=R_{T} \sqrt{2 / E_{i}}$ where $E_{i}$ is the incident energy in $\mathrm{GeV}$ per nucleon. For lead+lead collisions at $200 \mathrm{GeV}$ per nucleon this gives $\tau=0.7 \mathrm{fm} / c$.

If we now assume a value of $\alpha_{s}$ of the order of $\frac{1}{3}$ in agreement with the conclusions of ref. [1] we find that the energy density produced by the time variation of the field is of the order of $1.2 \%$ of the energy density of the field. This is small compared to the value of $20 \%$ obtained in ref. [5] using Schwinger's mechanism. It should be noted however that while Schwinger's mechanism depends only on the field strength, eq. (13) contains a dependence on the incident energy. For instance if we consider the same lead +lead experiment for colliding beams with 200 $\mathrm{GeV}$ per nucleon the resulting energy density becomes $10 \%$ of the field energy. Dynamical pair production thus becomes comparable to Schwinger's mechanism for $\mathrm{CM}$ energies of the order of $1 \mathrm{TeV}$ per nucleon.

An interesting aspect of the energy dependence just mentioned is that it would also show up in the ratio of the production rates of quarks with different flavors. This can be seen by comparing the numbers of pairs produced per unit time and unit volume for different values of the fermion mass $m$. For $m=0$ this number is

$N=\frac{\alpha_{\mathrm{s}}}{12 \pi} \sigma_{0}^{2} \sqrt{\pi}\left[\left(R^{2} / 2+\tau_{0}^{2}\right)^{1 / 2}-\tau_{0}\right]$.

For a non vanishing value of the mass $m$ the corresponding formula is

$N=\frac{\alpha_{\mathrm{s}}}{12 \pi} \sigma_{0}^{2} R^{2} m^{2}\left(R^{2} / 2+\tau_{0}^{2}\right)^{1 / 2} I$,

where I is the following integral:

$$
\begin{aligned}
I= & \int_{2}^{\infty} x\left(1+2 / x^{2}\right)\left(1-4 / x^{2}\right)^{1 / 2} \\
& \times \exp \left(R^{2} m^{2} x^{2} / 2\right) \\
& \times\left[\exp \left(-y^{2}\right) / y-\sqrt{\pi} \operatorname{erfc}(y)\right] \mathrm{d} x,
\end{aligned}
$$

with $y=m x\left(\tau_{0}^{2}+R^{2} / 2\right)^{1 / 2}$.

When $y / x \gg 1$ (i.e. $m R \gg 1$ ) and $2 m \tau_{0} \gg 1$ eq. (16) simplifies to

$N \simeq \frac{\alpha_{\mathrm{s}}}{512 \sqrt{\pi}} \frac{\sigma_{0}^{2}}{2} \frac{R^{2}}{\left(R^{2} / 2+\tau_{0}^{2}\right) m} \frac{\exp \left(-4 m^{2} \tau_{0}^{2}\right)}{\left(m \tau_{0}\right)^{3}}$,

while in the limit $m R \gg 1$ and $2 m \tau_{0} \ll 1$ it becomes

$N \simeq \frac{3 \alpha_{\mathrm{s}}}{156} \frac{\sigma_{0}^{2}}{2} \frac{R^{2}}{\left(R^{2} / 2+\tau_{0}^{2}\right) m}$.

The approximate expressions (18) and (19) are well 
adapted to calculate the production of charmed quarks since in this case $m_{\mathrm{c}}=6.75 \mathrm{fm}^{-1}[11,12]$. As an example let us calculate the ratio $N_{\mathrm{c}} / N_{\mathrm{u}}$ for lead+lead collisions at $400 \mathrm{GeV}$ per nucleon in the laboratory system. Using eq. (18) with $\tau_{0} \simeq 0.5 \mathrm{fm}^{-1}$, we obtain $N_{\mathrm{c}} / N_{\mathrm{u}} \simeq 3 \times 10^{-24}$. It is interesting to note that larger values would be obtained at higher energies. For instance $N_{\mathrm{c}} / N_{\mathrm{u}}=0.05$ for colliding lead beams at $300 \mathrm{GeV}$ per nucleon. It is also interesting to note that in the Schwinger mechanism the ratio $N_{\mathrm{c}} / N_{\mathrm{u}}$ does not depend on $\tau_{0}$ i.e. on the incident energy but only on the chromoelectric field strength $E$. It is given by

$\frac{N_{\mathrm{c}}}{N_{\mathrm{u}}} \simeq \frac{6}{\pi^{2}} \exp \left(-\pi m_{\mathrm{c}}^{2} / e E\right)$

which is $2 \times 10^{-13}$ for a field strength $E \simeq 5 \mathrm{fm}^{-2}$ as used in ref. [4]. A more detailed discussion of the ratio $N_{\mathrm{c}} / N_{\mathrm{u}}$ can be found in ref. [13].

In conclusion we have seen that dynamical pair production may become comparable to Schwinger's mechanism in nuclear collisions at high energies. A consequence of this mechanism is that it would lead to an important energy dependence of the ratio between the production rate for heavy flavors to that of light flavors. This effect should thus definitely be kept in mind when discussing strangeness production as- sociated with the possible production of a quarkgluon plasma.

We wish to thank J.P. Blaizot, C. Gerschell, J. Hüfner and T. Matsui for helpful discussions. One of the authors (C. Martin) wishes to thank the Direction des Recherches et Etudes Techniques for financial support.

\section{References}

[1] F.E. Low, Phys. Rev. D 12 (1975) 163.

[2] S. Nussinov, Phys. Rev. Lett. 34 (1975) 1286.

[3] T.S. Biro, H.B. Nielsen and J. Knoll, Nucl. Phys. B 245 (1984) 449;

A. Bialas and W. Czyz, Phys. Rev. D 31 (1985) 198; Nucl. Phys. B 267 (1986) 242.

[4] A.K. Kerman, T. Matsui and B. Svetitsky, Phys. Rev. Leti. 56 (1986) 219.

[5] G. Gatoff, A.K. Kerman and T. Matsui, Phys. Rev. D 36 (1987) 114.

[6] J. Schwinger, Phys. Rev. 82 (1951) 664.

[7] E. Brezin and C. Itzykson, Phys. Rev. D 12 (1970) 1191.

[8] C. Itzykson and J.B. Zuber, Quantum field theory (McGrawHill, New York, 1980).

[9] R.C. Wang and C.Y. Wong, Phys. Rev. D 38 (1988) 348.

[10] C. Martin and D. Vautherin, Phys. Rev. D 38 (1988) 3593.

[11] C.A. Dominguez and E. de Rafael, Ann. Phys. (NY) 174 (1987) 372 .

[12] J. Gasser and H. Leutwyler, Phys. Rep. 87 (1982) 77.

[13] E.G. Gurvitch, Phys. Lett. B 87 (1979) 386. 